\title{
A Model for the Determination of the Resisting Torque of the Rolling Bearing Cage Motion of Slow-Speed Kinematic Pairs
}

\author{
PRZEMYSŁAW SZUMIŃSKI* and TOMASZ KAPITANIAK \\ Techical University of Lodz, Division of Dynamics, Stefanowskiego 1/15, 90-924 Lodz, Poland
}

(Received: 18 July 2003; accepted in revised form: 19 May 2004)

\begin{abstract}
A simplified, plane model for determination of resisting torques of the rolling bearing cage motion of low speed kinematic pairs is presented. The considerations are devoted to an arbitrary character of the point contact of a rolling element-cage pairs lying in a plane which is crossing the rolling elements in their geometrical centre. The model has been developed on the basis of the analysis of motion kinematics of a bearing track and rolling elements. The proposed algorithm allows for continuous tracking the resisting torque of the cage and its components during the kinematic pair motion.
\end{abstract}

Key words: Rolling bearing, Resisting torque to motion, Cage.

\section{Nomenclature.}

\begin{tabular}{|c|c|}
\hline$D$ & diameter of the rolling bearing groove $(\mathrm{m})$. \\
\hline$D_{m}$ & pitch diameter of the bearing $(\mathrm{m})$. \\
\hline$F_{k}$ & $\begin{array}{l}\text { resultant reaction between the } i \text {-th rolling element and the } \\
\text { cage }(N) \text {. }\end{array}$ \\
\hline$F_{k 1}, F_{k 2}$ & $\begin{array}{l}\text { reaction components (normal) between the rolling element and } \\
\text { the cage in the first and second contact point, respectively }(\mathrm{N})\end{array}$ \\
\hline$F_{k}^{t}$ & $\begin{array}{l}\text { total reaction force between the cage and the rolling element } \\
\text { assembly }(\mathrm{N}) \text {. }\end{array}$ \\
\hline$n_{k}$ & mass of the bearing cage $(\mathrm{kg})$. \\
\hline$M_{T k}$ & resisting torque of the rolling bearing cage motion $(\mathrm{N} \mathrm{mm})$. \\
\hline$p$ & number of contact points of the model under analysis $(-)$. \\
\hline$\dot{q}_{j-1}, \dot{q}_{j}$ & $\begin{array}{l}\text { angular velocity of the inner and outer bearing track, respec- } \\
\text { tively }(\mathrm{rad} / \mathrm{s}) \text {. }\end{array}$ \\
\hline$\dot{q}_{t}$ & $\begin{array}{l}\text { angular velocity vector of the rolling element around its own } \\
\text { axis of rotation }(\mathrm{rad} / \mathrm{s}) \text {. }\end{array}$ \\
\hline$r_{i}$ & radius of the $i$ th rolling element $(\mathrm{mm})$. \\
\hline$r_{k p}, g_{p}, D_{m p}$ & circumferential and radial geometrical quantities, $p=1,2$, see \\
\hline & $\begin{array}{l}\text { Figure } 1 \text {, of locations of the rolling element-cage pair contact } \\
\text { points }(\mathrm{mm}) \text {. }\end{array}$ \\
\hline & circumferential velocity vector of the rolling assembly ( $\mathrm{rad} / \mathrm{s})$. \\
\hline$T_{k 1}$ & $\begin{array}{l}\text { friction forces between the rolling element and the cage } i \\
\text { first and second contact point, respectively }(\mathrm{N}) \text {. }\end{array}$ \\
\hline
\end{tabular}

*Author for correspondence: e-mail: przeszum@p.lodz.pl 
$z \quad$ number of bearing rolling elements (-).

$\alpha_{l} \quad$ bearing operating angle (catalogue and real value is presented in Table 1) (rad).

$\chi$ angle between the direction of the vector of the resultant reaction force of the $i$-th rolling element-cage pair and the tangential to the pitch diameter of the bearing in the pitch centre of the rolling element (rad).

$\chi_{p}$ auxiliary angles of locations of the rolling element-cage pair contact points, $p=1,2$, see Figure 1, (rad).

$\gamma \quad$ angle between rolling elements (rad).

$\mu$ dynamic friction coefficient of the $i$ th rolling element-cage contact $(-)$, Table 1 .

$\theta_{p} \quad$ angles that determine the locations of the rolling element-cage pair contacts with respect to the rolling element pitch centre, $p=1,2$, see Figure 1, (rad).

\section{Introduction and Assumptions}

Generally, models of rolling bearings which can be found in literature can be divided in two groups. The first includes mathematical models which are based on numerical simulations of bearings behavior [1-3]. The second group includes an empirical and engineering models [4-6] which can be used in practical applications, also for diagnostics $[7,8]$. Difficulty is placed in connection of both above mentioned ways to utilize models working in real time. Variety of the bearings work characteristics and the bearings construction complicate formulation of an universal model. With regard to small importance of resistance to motion of the rolling elements-cage contact in most applications of rolling bearings lacks of any universal model. Beside, models which can be found in literature allow for approximation of resistance to motion of pair the rolling elements - cage. These methods are based on empirical coefficients connected with lubrication resistance [5,9], A procedure based on graph theory and matrix approach has been proposed in paper [10]. In this case the reliability evaluation and rolling element analysis is developed. High-speed ball bearings subjected to both axial and radial loads are investigated in paper [11]. Through the geometric analysis of a ball bearing and the force balance, several parameters can be easily obtained, like the normal forces acting on the contact points, the contact angle at either the inner or the outer raceways that vary with the bearing position angles. This allows analysing cage's angular velocity and thus the rolling elements-cage contact.

In the presented paper under assumptions taken below, formulation of simultaneous mathematical and practical models of resistance of the cage are shown. The main task of a rolling bearing is to transfer a radial or longitudinal force, whereas in order to maintain the bearing in motion, it is necessary to apply to it a moment of force balancing the resisting torque. The resistance to motion in a rolling bearing results, first of all, from the hysteresis of deformations of bearing elements, the character of an influence of a lubricating film, forging of bearing rolling elements in a lubricating medium, slides and microslides between rolling elements and bearing ring 
tracks caused by deformations, the geometry of contact, a pivoting motion of rolling elements, a slide between the bearing cage and rolling elements and contact seal slides in the case of sealed bearings. In turn, these phenomena depend generally on a design, kind and dimensions of a bearing, a value of loading and its direction, rotational speed, a kind and properties of the lubricating medium and the way of lubrication $[12,2,13]$. Taking into consideration the character of bearing system operation in actual kinematics pairs of robots, that is to say, short-term and repeatable lowspeed (low acceleration) motions, the basic factors that affect the magnitude of the resistance to motion in a bearing are rolling friction, viscous friction, the resistance to motion of the rolling elements-bearing cage assembly, and friction of contact seals in the case of sealed bearings. In the present paper, the resisting torque of the rolling bearing cage motion is to be defined, according to the assumption undertaken. In the presented considerations, it has been assumed that bearing rolling elements carry the load in the normal, direction to the cage surface. The contact between the cage and arbitrary rolling elements is assumed as a point contact. All contact points lie on plane which is crossing the rolling elements in their geometrical centre. A lack of deformations of the cage from the ideal circularity has been assumed as well. This assumption is the basis of limitations related to technical processes of the cage production $[12,13]$. It has been also assumed that rolling elements are symmetrically distributed on a track, they contact tracks in two opposite points at each instant and that rolling elements do not exhibit any significant error of shape. Assuming the motion takes place with low speed and low acceleration of kinematic pairs, it causes lubrication is of small importance on contact, [13], and temperature does not influence on the dimensions of the cage and rolling elements.

\section{Algorithm for Determination of the Cage Resisting Torque}

In this section a model has been presented and resisting torques of the bearing cage through an analysis of the rolling elements-cage contact of the rolling bearing have been determined. Dynamic friction between rolling elements and cage in the presented model is considered. The model does not include analysis of stiction. Cages of rolling bearings are carried on bearing rolling elements. In order to determine the reaction force and friction of the rolling element-cage contact, a linear contact between the $i$ th rolling element and the cage has been replaced by a point contact. The number of contact points depends on the cage design, i.e., on the type of the cage-rolling element contact pair. Taking into account the shape of cross-sections of cages used in rolling bearings, a two-point model of contact of the rolling element with the cage has been assumed (Figure 1). This assumption allows simplifying the model for practical application. The presented theory concerns an arbitrary number of contact points of the rolling element-cage pair model, including a one-point contact.

In Figure 1 the notations, which are introduced into the proposed model, can be found. Symbols are given in Nomenclature. The friction force between a rolling element and a cage will appear at the moment that the angular velocity vector of the rolling element around its own axis of rotation $\dot{q}_{t}$, equation (3), and the circumferential velocity vector of the rolling assembly $u_{m}$, equation (4), reach values different 


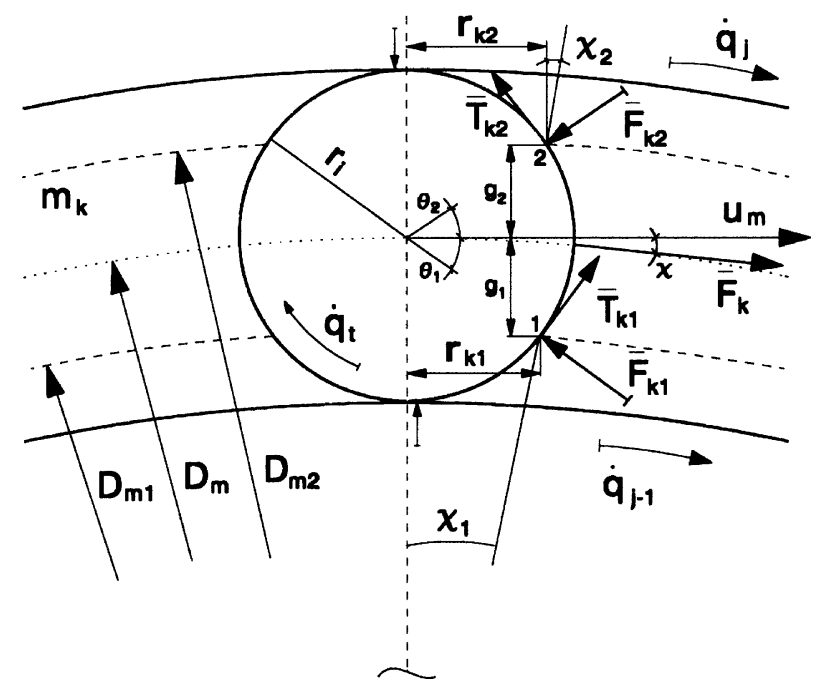

Figure 1. Schematic view of the model of a two-point contact between the rolling element-cage pair of the rolling bearing.

from zero. In order for the reaction forces between a rolling element and a cage to occur, it is sufficient when the circumferential velocity vector of the rolling assembly $u_{m}$ reaches a value different from zero. The angular velocity of the rolling element assembly with respect to the inner ring can be expressed by, [13] and Figure 1,

$$
\dot{q}_{m, j-1}=\dot{q}_{m}-\dot{q}_{j-1}=\frac{1}{2}\left(\dot{q}_{j}-\dot{q}_{j-1}\right) \cdot\left(1+\frac{2 \cdot r_{i} \cdot \cos \left(\alpha_{l}\right)}{D_{m}}\right)
$$

The circumferential velocity of the rolling part is the same as the track velocity and thus it can be written as

$$
\dot{q}=\left(\frac{D_{m}-2 \cdot r_{i} \cdot \cos \left(\alpha_{l}\right)}{2 \cdot r_{i}}\right) \cdot \dot{q}_{m, j-1}
$$

Finally, the angular velocity of the rolling element around its own axis equals:

$$
\dot{q}=\frac{D_{m}}{4 \cdot r_{i}} \cdot\left(\dot{q}_{j}-\dot{q}_{j-1}\right) \cdot\left(1-\left(\frac{2 \cdot r_{i} \cdot \cos \left(\alpha_{l}\right)}{D_{m}}\right)^{2}\right)
$$

The relationship describing the circumferential velocity of the rolling element centre is as follows, [13,14],

$$
u_{m}=\frac{D_{m}}{4} \cdot\left[\dot{q}_{j-1} \cdot\left(1-\frac{2 \cdot r_{i} \cdot \cos \left(\alpha_{l}\right)}{D_{m}}\right)+\dot{q}_{j} \cdot\left(1+\frac{2 \cdot r_{i} \cdot \cos \left(\alpha_{l}\right)}{D_{m}}\right)\right]
$$

The friction force between the $i$-th rolling element and the cage is equal to the sum of friction forces acting in contact points between the rolling element and the cage, (Figure 1),

$$
\bar{T}_{k}=\sum_{p} \bar{T}_{k p} \equiv \bar{T}_{k 1}+\bar{T}_{k 2}=\mu \cdot \bar{F}_{k 1}+\mu \cdot \bar{F}_{k 2}
$$


where symbols are given in Nomenclature. Dynamic coefficient of friction $\mu$ can be assumed to vary in individual contact points.

Owing to variability in the radii along which the reaction forces that result from cage designs act, values of friction component forces can depend on, among others, the direction of the relative rotation of the outer and inner tack. The value of the resultant reaction force acting between the cage and the $i$ th rolling element, obtained as a result of the sum of projections of the forces acting on this element on the tangential to the bearing pitch diameter in the pitch centre of the rolling element, is equal to (see Figure 1 and Appendix),

$$
\begin{aligned}
F_{k} \cdot \cos (\chi)=\sum_{p}\left(F_{k p}^{t}+T_{k p}^{t}\right) \equiv & F_{k 1} \cdot\left[\cos \left(\theta_{1}\right)-\operatorname{sgn}\left(\dot{q}_{t}\right) \cdot \operatorname{sgn}\left(u_{m}\right) \cdot \mu \cdot \sin \left(\theta_{1}\right)\right] \\
& +F_{k 2} \cdot\left[\cos \left(\theta_{2}\right)+\operatorname{sgn}\left(\dot{q}_{t}\right) \cdot \operatorname{sgn}\left(u_{m}\right) \cdot \mu \cdot \sin \left(\theta_{2}\right)\right]
\end{aligned}
$$

as a result of the projection on the normal axis

$$
\begin{aligned}
F_{k} \cdot \sin (\chi)=\sum_{p}\left(F_{k p}^{n}+T_{k p}^{n}\right) \equiv & F_{k 1} \cdot\left[\sin \left(\theta_{1}\right)+\operatorname{sgn}\left(\dot{q}_{t}\right) \cdot \operatorname{sgn}\left(u_{m}\right) \cdot \mu \cdot \cos \left(\theta_{1}\right)\right] \\
& -F_{k 2} \cdot\left[\sin \left(\theta_{2}\right)-\operatorname{sgn}\left(\dot{q}_{t}\right) \cdot \operatorname{sgn}\left(u_{m}\right) \cdot \mu \cdot \sin \left(\theta_{2}\right)\right]
\end{aligned}
$$

The angles $\theta_{p}, \chi$ and the lengths $g_{p}$ (for $p=1,2$ ), are generated below on the basis of the trigonometric analysis of the respective triangles presented in Figure 1.

\subsection{Determination of Geometrical Quantities $g_{p}$ as a Function of the}

Rolling Element Radius and the Pitch Diameter of the Bearing and the Contact Point of the Rolling element-Cage Assembly

The geometrical and analytical relationships that allow the determination of the quantity $g_{2}$ are shown in Figure 2(a) and in the form of the following equations:

$$
\begin{gathered}
r_{k 2}^{2}+g_{2}^{2}=r_{i}^{2} \\
r_{k 2}^{2}+\left(g_{2}+\frac{D_{m}}{2}\right)^{2}=\left(\frac{D_{m_{2}}}{2}\right)^{2}
\end{gathered}
$$
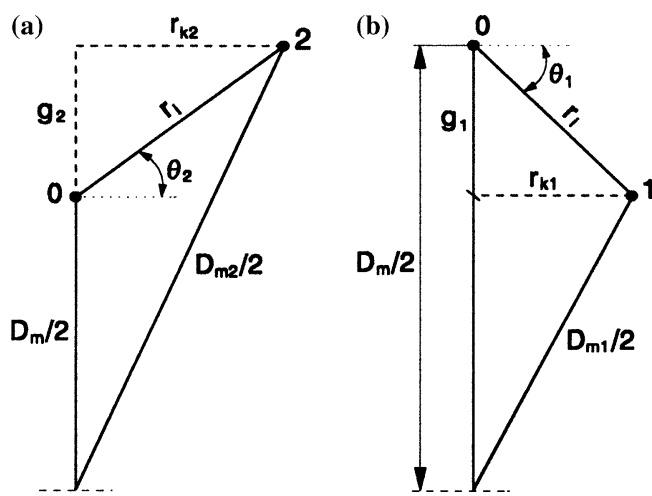

Figure 2. Determination of the geometrical relations for determination of the quantities $g_{p}, p=1,2$. 


\section{Przemyslaw Szumiński and Tomasz Kapitaniak}

and correspondingly, for the case of determination of the quantity $g_{1}-$ in Figure $2 \mathrm{~b}$ and in the form of the following equations:

$$
\begin{gathered}
r_{k 1}^{2}+g_{1}^{2}=r_{i}^{2} \\
r_{k 1}^{2}+\left(\frac{D_{m}}{2}-g_{1}\right)^{2}=\left(\frac{D_{m_{1}}}{2}\right)^{2}
\end{gathered}
$$

Sets of equations (8) and (9) have been written in the general form

$$
\begin{gathered}
r_{k p}^{2}+g_{p}^{2}=r_{i}^{2} \\
r_{k p}^{2}+\left(\frac{D_{m}}{2}+\rho \cdot g_{p}\right)^{2}=\left(\frac{D_{m p}}{2}\right)^{2}
\end{gathered}
$$

where $p=1,2, \rho=-1<=>p=1, \rho=1<=>p=2$.

While determining the quantity $g_{p}(p=1,2)$ from sets of equations (8) and (9), we have obtained

$$
g_{p}=\rho \cdot\left(\frac{\left(\frac{D_{m}}{2}\right)^{2}-\left(\frac{D_{m p}}{2}\right)^{2}+r_{i}^{2}}{D_{m}}\right)
$$

where $p=1(\rho=1), 2(\rho=-1)$.

\subsection{Determination of the Angle $\chi$ of the Reaction Force $\bar{F}_{k}$ Direction}

Analysing Figure 3, we can write the following relationships of geometrical quantities:

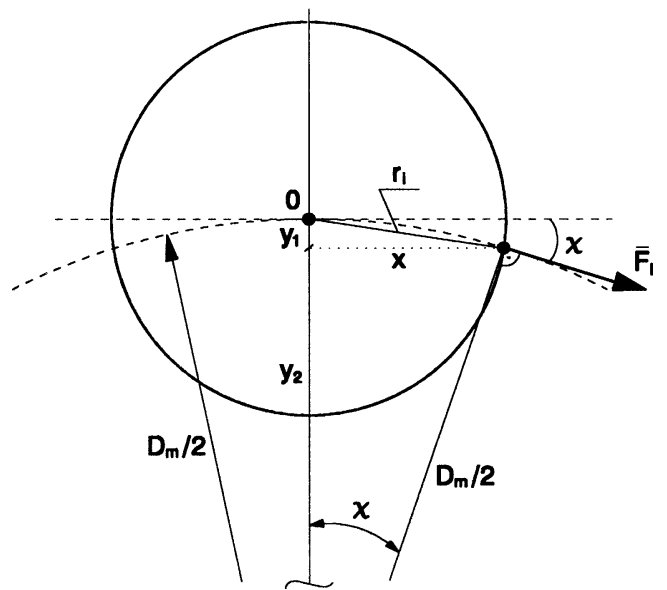

Figure 3. Determination of the angle $\chi$ of the resultant reaction $F_{k}$, Figure 1. 


$$
\begin{aligned}
x^{2}+y_{1}^{2} & =r_{i}^{2} \\
x^{2}+y_{2}^{2} & =\left(\frac{D_{m}}{2}\right)^{2} \\
y_{1}+y_{2} & =\frac{D_{m}}{2}
\end{aligned}
$$

Extracting the first two equations of equations (12) from each other and employing the third equation from this set of equations, through determination of the quantity $y_{1}$ and its substitution into the obtained difference of the first two equations, we obtain

$$
y_{2}=\frac{\frac{1}{2} \cdot D_{m}^{2}-r_{i}^{2}}{D_{m}}
$$

On the basis of Figure 3, we can write

$$
\cos (\chi)=\left(\frac{2 \cdot y_{2}}{D_{m}}\right)
$$

The angle $\chi$ of the resultant reaction force $\bar{F}_{k}$ of the rolling element-cage pair is then equal to

$$
\chi=\mathrm{a} \cos \left(1-\frac{2 \cdot r_{i}^{2}}{D_{m}^{2}}\right)
$$

Eventually, the angles $\theta_{p}, \chi$ and the lengths $g_{p}$ are expressed as follows:

$$
\begin{array}{r}
\theta_{p}=\mathrm{a} \sin \left(\frac{g_{p}}{r^{i}}\right), \quad \chi=\mathrm{a} \cos \left(1-\frac{2 \cdot r_{i}^{2}}{D_{m}^{2}}\right) \\
g_{p}=\rho \cdot\left(\frac{\left(\frac{D_{m}}{2}\right)^{2}-\left(\frac{D_{m p}}{2}\right)^{2}+r_{i}^{2}}{D_{m}}\right)
\end{array}
$$

where $p=1(\rho=1), 2(\rho=-1)$.

In the general case, the motion of the $i$ th rolling element in the time interval $\Delta t$ is non-uniformly variable. The total circumferential reaction force between the rolling elements and the cage has been expressed by the following relationship:

$$
\begin{array}{r}
\text { if } \Delta u_{m}=u_{m}(t+\Delta t)-u_{m}(t) \neq 0 \Rightarrow F_{k}^{t}=m_{k} \cdot\left(g+\left|a_{t}\right|\right) \\
\text { if } u_{m}(t)=\mathrm{const} \wedge u_{m}(t) \neq 0 \Rightarrow F_{k}^{t}=m_{k} \cdot g \\
\text { if } u_{m}(t)=0 \wedge \dot{u}_{m}(t)=0 \Rightarrow F_{k}^{t}=0 \\
a_{t}=\left.\frac{\mathrm{d} u_{m}}{\mathrm{~d} t}\right|_{t}=\left.\lim _{\Delta_{t} \rightarrow 0} \frac{\Delta u_{m}}{\Delta t} \cong \frac{\Delta u_{m}}{\Delta t}\right|_{\Delta t \rightarrow 0}
\end{array}
$$

where $\Delta t=\left(t_{i}-t_{i-1}\right)$ is the time interval of the motion simulation [s], and $a_{t}$ is the acceleration of an arbitrary rolling element (also a cage) determined at the instant $t\left(\mathrm{~m} / \mathrm{s}^{2}\right)$. Depending on the fact if the analytical form of the angular velocity of the 


\section{Przemyslaw Szumiński and Tomasz Kapitaniak}

bearing track $\dot{q}_{j-1}, \dot{q}_{j}$ (in the case of the bearing under analysis, the relative velocity of the bearing track in the form of equation (25) has been assumed) and the linear velocity $u_{m}$ of the rolling element are known or only the numerical form, we can use different relationships in order to determine the acceleration $a_{t}\left(a_{t}\right.$ in equation (17)). In the case of a uniform motion of the $i$ th rolling element in the time interval $\Delta t\left(\Delta u_{m}=0\right)$, the reaction force between the cage and the rolling element at the instant $t+\Delta t$ and at the instant $t$ are equal $F_{k}^{t}(t+\Delta t)=F_{k}^{t}(t)$.

The equation of moments of reaction forces and moments of friction acting between $i$ th rolling element and the cage with respect to the bearing axis (see Appendix for understanding signs of the particular terms) has the form

$$
\begin{aligned}
M_{T k}^{i}= & \Sigma_{p} M_{T k p}^{i} \equiv F_{k 1} \cdot\left[r_{k 1} \cdot \sin \left(\theta_{1}\right)+\frac{D_{m 1}}{2} \cdot \cos \left(\theta_{1}\right) \cdot \cos \left(\chi_{1}\right)+\operatorname{sgn}\left(\dot{q}_{t}\right) \cdot \operatorname{sgn}\left(u_{m}\right) \cdot\right. \\
& \left.\mu \cdot\left[r_{k 1} \cdot \cos \left(\theta_{1}\right)-\frac{D_{m 1}}{2} \cdot \sin \left(\theta_{1}\right) \cdot \cos \left(\chi_{1}\right)\right]\right]+F_{k 2} \cdot\left[\frac{D_{m 2}}{2} \cdot \cos \left(\theta_{2}\right) \cdot \cos \left(\chi_{2}\right)\right. \\
& \left.-r_{k 2} \cdot \sin \left(\theta_{2}\right)+\operatorname{sgn}\left(\dot{q}_{1}\right) \cdot \operatorname{sgn}\left(u_{m}\right) \cdot \mu \cdot\left[r_{k 2} \cdot \cos \left(\theta_{2}\right)-\frac{D_{m 2}}{2} \cdot \sin \left(\theta_{2}\right) \cdot \cos \left(\chi_{2}\right)\right]\right]
\end{aligned}
$$

whereas

$$
\chi_{p}=\mathrm{a} \sin \left(\frac{2 \cdot r_{k p}}{D_{m p}}\right)=\mathrm{a} \cos \left(\frac{D_{m}-2 \cdot \rho \cdot g_{p}}{D_{m p}}\right)
$$

where $\rho=1(\rho=1), 2(\rho=2)$. Symbols are given in Nomenclature. The quantities $r_{i}, D_{m}, D_{m p}$ are treated as geometrical data of the bearing (their values can depend of the sense of the circumferential velocity vector of the rolling bearing assembly $u_{m}$ ).

The geometrical quantities $r_{k p}$ occurring in the above-mentioned equation have been determined from the following relation, see Figure 1:

$$
r_{k p}=\sqrt{r_{i}^{2}-g_{p}^{2}}
$$

where $\rho=1,2$.

Employing relation (6), the equation of moments of the forces acting between the rolling elements and the cage with respect to the bearing axis, assumes the form

$$
\begin{aligned}
M_{T k}= & \Sigma_{z}\left[F _ { k } \cdot \operatorname { c o s } ( \chi ) \cdot \left(\frac{D_{m 1}}{2} \cdot \cos \left(\chi_{1}\right)\right.\right. \\
& \left.+\frac{r_{k 1} \cdot \sin \left(\theta_{1}\right)+\operatorname{sgn}\left(\dot{q}_{t}\right) \cdot \operatorname{sgn}\left(u_{m}\right) \cdot \mu \cdot r_{k 1} \cdot \cos \left(\theta_{1}\right)}{\cos \left(\theta_{1}\right)-\operatorname{sgn}\left(\dot{q}_{t}\right) \cdot \operatorname{sgn}\left(u_{m}\right) \cdot \mu \cdot \sin \left(\theta_{1}\right)}\right) \\
& +F_{k 2} \cdot\left[\left(\cos \left(\theta_{2}\right)+\operatorname{sgn}\left(\dot{q}_{1}\right) \cdot \operatorname{sgn}\left(u_{m}\right) \cdot \mu \cdot \sin \left(\theta_{2}\right)\right) \cdot\right. \\
& \left(\frac{D_{m 2}}{2} \cdot \cos \left(\chi_{2}\right)-\frac{D_{m 1}}{2} \cdot \cos \left(\chi_{1}\right)-r_{k 1} \cdot \sin \left(\theta_{1}\right) \cdot\right. \\
& \left.\frac{1+\operatorname{sgn}\left(\dot{q}_{t}\right) \cdot \operatorname{sgn}\left(u_{m}\right) \cdot \mu \cdot \tan ^{-1}\left(\theta_{1}\right)}{\cos \left(\theta_{1}\right)-\operatorname{sgn}\left(\dot{q}_{t}\right) \cdot \operatorname{sgn}\left(u_{m}\right) \cdot \mu \cdot \sin \left(\theta_{1}\right)}\right) \\
& \left.\left.+r_{k 2} \cdot \sin \left(\theta_{2}\right) \cdot\left(\operatorname{sgn}\left(\dot{q}_{t}\right) \cdot \operatorname{sgn}\left(u_{m}\right) \cdot \mu \cdot \tan ^{-1}\left(\theta_{2}\right)-1\right)\right]\right]
\end{aligned}
$$


whereas, employing relations (6) and (7), we can present the force $F_{k 2}$ as follows:

$$
F_{k 2}=F_{k} \cdot \frac{\cos (\chi) \cdot\left[\sin \left(\theta_{1}\right)+\operatorname{sgn}\left(\dot{q}_{t}\right) \cdot \operatorname{sgn}\left(u_{m}\right) \cdot \mu \cdot \cos \left(\theta_{1}\right)\right]-\sin (\chi) \cdot\left[\cos \left(\theta_{1}\right)-\operatorname{sgn}\left(\dot{q}_{t}\right) \cdot \operatorname{sgn}\left(u_{m}\right) \cdot \mu \cdot \sin \left(\theta_{1}\right)\right]}{\left[1+\operatorname{sgn}^{2}\left(\dot{q}_{t}\right) \cdot \operatorname{sgn}^{2}\left(u_{m}\right) \cdot \mu^{2}\right] \cdot\left[\sin \left(\theta_{2}\right) \cdot \cos \left(\theta_{1}\right)+\sin \left(\theta_{1}\right) \cdot \cos \left(\theta_{2}\right)\right]}
$$

Having substituted relation (22) into (21), we finally obtain

$$
\begin{aligned}
& M_{T k}=\sum_{z} \\
& {\left[F _ { k } \cdot \operatorname { c o s } ( \chi ) \cdot \left[\frac{D_{m 1}}{2} \cdot \cos \left(\chi_{1}\right)+\frac{r_{k 1} \cdot \sin \left(\theta_{1}\right)+\operatorname{sgn}\left(\dot{q}_{t}\right) \cdot \operatorname{sgn}\left(u_{m}\right) \cdot \mu \cdot r_{k 1} \cdot \cos \left(\theta_{1}\right)}{\cos \left(\theta_{1}\right)-\operatorname{sgn}\left(\dot{q}_{t}\right) \cdot \operatorname{sgn}\left(u_{m}\right) \cdot \mu \cdot \sin \left(\theta_{1}\right)}\right.\right.} \\
&+\frac{\sin \left(\theta_{1}\right)+\operatorname{sgn}\left(\dot{q}_{t}\right) \cdot \operatorname{sgn}\left(u_{m}\right) \cdot \mu \cdot \cos \left(\theta_{1}\right)-\tan (\chi) \cdot\left[\cos \left(\theta_{1}\right)-\operatorname{sgn}\left(\dot{q}_{t}\right) \cdot \operatorname{sgn}\left(u_{m}\right) \cdot \mu \cdot \sin \left(\theta_{1}\right)\right]}{\left(1+\operatorname{sgn}^{2}\left(\dot{q}_{t}\right) \cdot \operatorname{sgn}^{2}\left(u_{m}\right) \cdot \mu^{2}\right) \cdot\left(\sin \left(\theta_{2}\right) \cdot \cos \left(\theta_{1}\right)+\sin \left(\theta_{1}\right) \cdot \cos \left(\theta_{2}\right)\right)} \\
& \times\left[\left(\cos \left(\theta_{2}\right)+\operatorname{sgn}\left(\dot{q}_{t}\right) \cdot \operatorname{sgn}\left(u_{m}\right) \cdot \mu \cdot \sin \left(\theta_{2}\right)\right)\right. \\
&\left(\frac{D_{m 2}}{2} \cdot \cos \left(\chi_{2}\right)-\frac{D_{m 1}}{2} \cdot \cos \left(\chi_{1}\right)-r_{k 1} \cdot \sin \left(\theta_{1}\right)\right. \\
&\left.\quad \times \frac{1+\operatorname{sgn}\left(\dot{q}_{t}\right) \cdot \operatorname{sgn}\left(u_{m}\right) \cdot \mu \cdot \tan ^{-1}\left(\theta_{1}\right)}{\cos \left(\theta_{1}\right)-\operatorname{sgn}\left(\dot{q}_{t}\right) \cdot \operatorname{sgn}\left(u_{m}\right) \cdot \mu \cdot \sin \left(\theta_{1}\right)}\right)+r_{k 2} \cdot \sin \left(\theta_{2}\right) \\
&\left.\left.\left.\left(\operatorname{sgn}\left(\dot{q}_{t}\right) \cdot \operatorname{sgn}\left(u_{m}\right) \cdot \mu \cdot \tan ^{-1}\left(\theta_{2}\right)-1\right)\right]\right]\right]
\end{aligned}
$$

where the force $F_{k}$ is determined from equation (17) as $F_{k}=\frac{F_{k}^{t}}{Z}$, according to Nomenclature.

In the case of a one-point contact between the $i$ th rolling element and the cage, as a result of the simplification of the above-mentioned formulas, the resisting torque of the cage takes the form

$$
\begin{aligned}
M_{T k}= & \sum_{z}\left[F_{k} \cdot \cos (\chi) \cdot\right. \\
& \left.\left(\frac{D_{m 1}}{2} \cdot \cos \left(\chi_{1}\right)+\frac{r_{k 1} \cdot \sin \left(\theta_{1}\right)+\operatorname{sgn}\left(\dot{q}_{t}\right) \cdot \operatorname{sgn}\left(u_{m}\right) \cdot \mu \cdot r_{k 1} \cdot \cos \left(\theta_{1}\right)}{\cos \left(\theta_{1}\right)-\operatorname{sgn}\left(\dot{q}_{t}\right) \cdot \operatorname{sgn}\left(u_{m}\right) \cdot \mu \cdot \sin \left(\theta_{1}\right)}\right)\right]
\end{aligned}
$$

The quantities and data of bearing which are necessary for execution of calculations are specified in Table 1.

\section{An Example of the Analysis of Resisting Torque of the Rolling Elements-Cage Contact of a Low-Speed Kinematic Pair}

In order to present an example of the numerical analysis, a $7206 B E$ angular ball bearing has been considered. Manipulators of industrial robots perform usually periodic motions. Therefore, the kinematic of motion of the kinematic pair is periodically variable. This periodicity can be approximated with harmonic functions. Thus, in order to conduct an exemplary simulation, the relative velocity of the rotational 


\section{Przemystaw Szumiński and Tomasz Kapitaniak}

motion between the inner and outer bearing track, have been assumed in the form

$$
\dot{q}=G \cdot[\eta-\sin (\omega \cdot t)]
$$

where $G$ is the relative velocity amplitude, $\eta$ denotes the parameter of the bearing motion character, whereas $\omega$ is the angular velocity of changes in the relative motion. Figures 4 and 5 have been drawn assuming the following values of kinematics parameters: $G=10 \mathrm{rad} / \mathrm{s}, \eta=2, \omega=15 \mathrm{rad} / \mathrm{s}$. A zero radial clearance and a zero value of the angular velocity of the inner bearing ring have been assumed as well.

The values referring to dynamic dry friction coefficients $\mu$ are quoted after $[15$, 16]. The remaining, lacking data concerning the $7206 B E$ bearing are to be found in [9].

Some sample results of the numerical simulations are shown in Figures 4 and 5 . Figure 4 illustrates a resultant reaction force between the $i$ th rolling element and the cage. In Figure 5, a sample resisting torque of the rolling elements-cage contact motion as a function of time and bearing track relative velocity, equation (25), have been presented. The data of the rolling bearing under analysis are presented in Table 1.

Presented results can be compared with approximated data presented in $[5,13]$. Comparison is possible by appointing another type of components of resistance to the motion. Subtraction of components of resistance to motion from total resistance (which can be found in the literature in form of empirical coefficients) allows to approximate the value of resistance of the rolling elements-cage motion. This approach allows to found general differences which do not exceed $10 \%$ in the considered case and follow particular assumptions [5,13,17]. Presented model is a part of the algorithm for estimation of the resistance in rolling kinematic pairs of rotary type manipulators to the motion. The algorithm of this analysis can be found in [17].

\section{Conclusions}

A model of the analysis of the resisting torque of a low speed and low acceleration motion of the cage, based on the analysis of the rolling elements-cage pair contact of

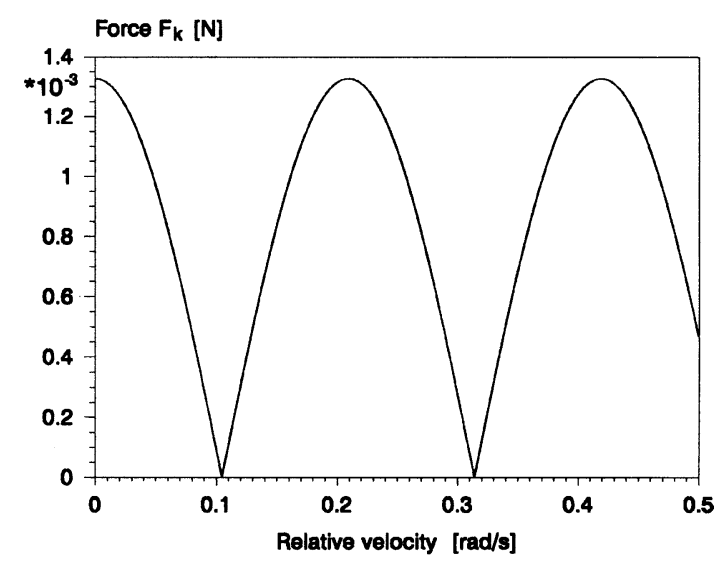

Figure 4. Resultant reaction force between the $i$ th rolling element and the cage. 


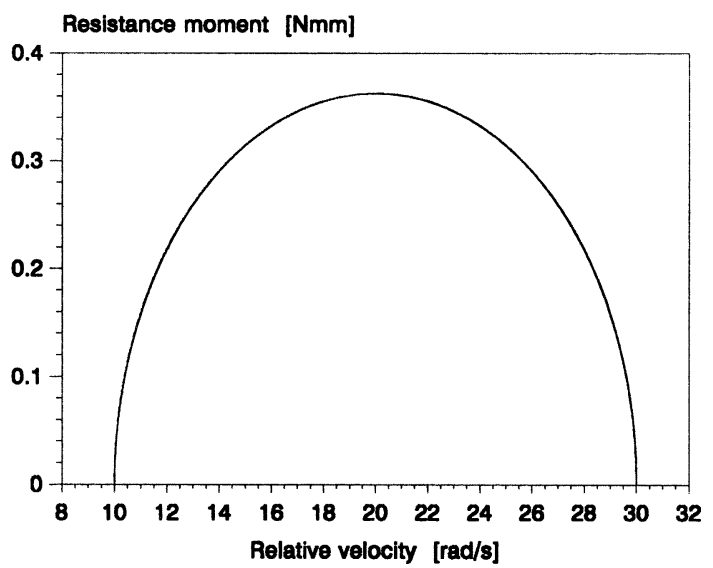

Figure 5. Resisting torque of the rolling elements-cage assembly.

Table 1 .

\begin{tabular}{ll}
\hline Number of rolling elements $\mathrm{z}(-)$ & 12 \\
\hline Dynamic friction coefficient $\mu(-)$ & 0.05 \\
\hline Rolling element radius $r_{i}(\mathrm{~m})$ & 0.00475 \\
\hline Diameter of the rolling bearing groove $D(\mathrm{~m})$ & 0.0365 \\
\hline Angle between rolling elements $\gamma\left({ }^{\circ}\right)$ & 30 \\
\hline Bearing angle $\alpha_{1}\left(^{\circ}\right)$ & $40 / 41.7$ \\
\hline Mass of the bearing cage $m_{k}(\mathrm{~kg})$ & 0.008 \\
\hline Pitch diameter $D_{m}(\mathrm{~m})$ & 0.046 \\
\hline Inner diameter $D_{m 1}(\mathrm{~m})$ & 0.043 \\
\hline
\end{tabular}

the rolling bearing, has been presented. The considerations refer to an arbitrary character of the point contact of the cage-rolling element pair. In the model it is assumed that all contact points lie in the plane crossing the rolling elements in their geometrical centre.

The algorithm allows for continuous tracing the resisting torque and its components in the form of the moment of friction and the moment of reaction of the rolling elements-cage assembly during operation of the low-speed and low acceleration kinematic pair. The presented model can be applied in analysis of resistance to motion of rolling kinematic pairs of manipulators. Its advantage is a possibility of easy application.

\section{Acknowledgements}

The investigations has been supported by the Polish Scientific Research Committee (KBN) under project 5T07A00525. 

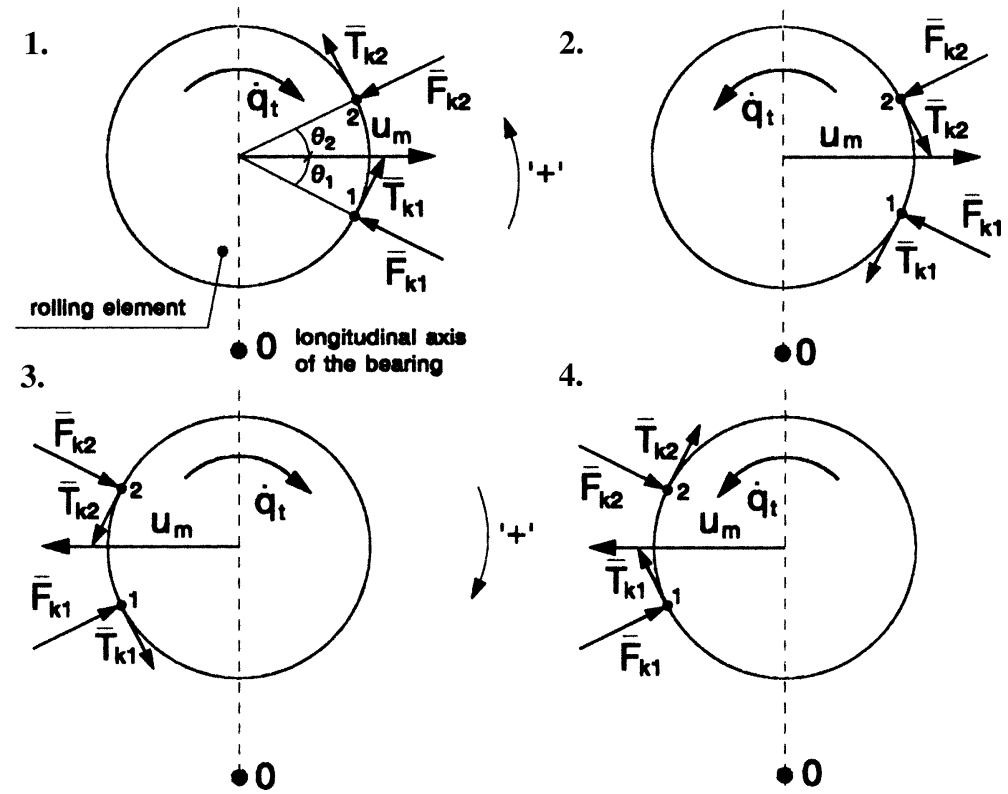

Figure 6. Reaction forces of the rolling element-cage assembly of the rolling bearing. The quantities $u_{m}$ and $\dot{q}_{t}$ are expressed by Equations (4) and (3), correspondingly.

\section{Appendix}

In Figure 6, an analysis of senses of component forces and of moments of reaction and friction of the rolling element-cage assembly with respect to the longitudinal axis ' 0 ' of the bearing is shown.

Table 2 presents an analysis of components of reaction and friction forces of the rolling element-cage assembly of the rolling bearing.

Table 2.

\begin{tabular}{ccccc}
\hline$\Sigma\left(F_{k p}, T_{k p}, M_{i 0}\right):$ & 1 & 2 & 3 & 4 \\
\hline$F_{k 1} \cdot \sin \left(\theta_{1}\right)$ & + & + & + & + \\
$F_{k 1} \cdot \sin \left(\theta_{2}\right)$ & - & - & - & - \\
$F_{k 1} \cdot \cos \left(\theta_{1}\right)$ & + & + & + & + \\
$F_{k 1} \cdot \cos \left(\theta_{2}\right)$ & + & + & + & + \\
$T_{k 1} \cdot \cos \left(\theta_{1}\right)$ & + & - & - & + \\
$T_{k 1} \cdot \sin \left(\theta_{1}\right)$ & - & + & + & - \\
$T_{k 1} \cdot \cos \left(\theta_{2}\right)$ & + & - & - & + \\
$T_{k 1} \cdot \sin \left(\theta_{2}\right)$ & + & - & - & + \\
\hline
\end{tabular}

The form, in which the senses of components of forces and moments of forces as a function of the kinematics of the rolling elements and track motion, shown in Equations (6), (7) and (18), results from the analysis that has been carried out. 


\section{References}

1. Bordon, A., Rigal, J.F. and Play, D., 'Static rolling bearing models in CAD environment for the study complex mechanisms, Part 1-rolling bearing model', ASME J.Tribol. 121(2) (1999) 205-214.

2. Houpert, L., 'A uniform analytical approach for ball and roller bearings calculations', $A S M E$ J. Tribol. 119(4), (1997), 851-858.

3. Fritzsona, D. and Nordling, P., 'Adaptive scheduling strategy optimizer for parallel rolling bearing simulation', Future Generation Computer Systems 16(5), (2000), 563-570.

4. Brandlein, J. and Hasbargen, L., Ball and Roller Bearings - Theory, Design and Application, John Wiley, (1999).

5. Harris, T.A., Rolling Bearing Analysis, John Wiley, 2000.

6. McGowan, K., Bearings-plain, Ball and Roller: Competitive Analysis, World Information Technologies, 1993.

7. Lynagha, N., Rahnejat, H., Ebrahimia, M. and Ainib, R., 'Bearing induced vibration in precision high speed routing spindles', Int. J. Mach. Tools Manuf. 40(4), (2000), 561-577.

8. Tandon, N. and Choudhury, A., 'A review of vibration and acoustic measurement methods for the detection of defects in rolling element bearings', Tribol. Int., 32(8), (1999), 469-480.

9. SKF - Main catalogue.

10. Sehgala, R., Gandhi, O.P. and Angrac, S., 'Reliability evaluation and selection of rolling element bearings', Reliab. and Eng. Syst. Saf. 68(1), (2000), 39-52.

11. Neng Tung Liaoa and Lin, J. F., 'Ball bearing skidding under radial and axial loads', Mech. Mach Theory 37(1), (2002), 91-113.

12. Eschmann, P., Hasbargen, L. and Weigand, K., Ball and Roller Bearings - Theory, Design and Application, John Wiley, 1985.

13. Krzemiński-Freda, H., Rolling Bearings, State Scientific Publishers, Warsaw, Poland, 1989.

14. Szumiński, P., 'Determination of the dynamic angle of the bearing rolling element location and of its influence on the loads and rolling friction', Int. J. Robot. Sys. 17(12), (2000), 671-687.

15. Szczeniowski, Sz., Experimental Physics: Mechanics and Acoustics, State Scientific Publishers, Warsaw, Poland, 1972.

16. Manual for Mechanical Engineers, State Scientific Publishers, Warsaw, Poland, 1991.

17. Szumiński, P. and Kapitaniak, T., 'Analysis of resisting torques of rolling kinematic pairs of robots', Proceedings of Fifth International Symposium on Methods and Models in Automation and Robotics, Miedz'yzdroje, Poland, (1998), 951-956. 